\title{
Early Feeding of a High-Cholesterol Diet Enhances Intestinal Permeability to Lipids in Rabbits
}

\author{
A. B. R. THOMSON, M. KEELAN, AND M. TAVERNINI
}

Nutrition and Metabolism Research Group, University of Alberta, Edmonton, Alberta, Canada

\begin{abstract}
This study was undertaken in young and growing rabbits to establish the of feeding a high- $(2 \%)$ cholesterol diet (HC) on the intestinal in vitro uptake of a homologous series of saturated fatty acids, dodecanol, cholesterol, and bile acids. In the jejunum there was an increase in the uptake of myristic acid and dodecanol in HC as compared with animals fed Purina Chow, whereas in the ileum the uptake of stearic acid, cholesterol, and taurocholic acid was reduced. There was increased jejunal uptake of medium-chain length fatty acids, increased incremental change in free energy $\left(\int \Delta \mathrm{Fw} \rightarrow l\right)$, increased uptake of palmitic, taurocholic acid, and glycocholic acid and reduced uptake of myristic acid and dodecanol in animals fed the high-cholesterol diet for $\mathbf{1 0}$ days followed by Purina Chow for 18 days (HC-chow), as compared with HC. There was also increased ileal uptake of palmitic acid in $\mathrm{HC}$-chow as compared with $\mathrm{HC}$ but reduced ileal uptake of stearic acid, cholesterol, taurocholic acid, and glycocholic acid. In the colon, there was increased uptake of stearic acid and dodecanol in HC-C as compared with $\mathrm{HC}$, but reduced uptake of cholesterol, taurocholic acid and glycocholic acid. These changes in lipid uptake were not explained by alterations in the mucosal surface area or in the animals' body weight gain. It is proposed that 1) early feeding experiences with a high-cholesterol diet may alter the normal development of intestinal transport of lipids with increased jejunal permeability to medium-chain fatty acids and increased effective resistance of the intestinal unstirred water layer; and 2) this effect of the high-cholesterol diet on the uptake of some lipids may not become manifest until the animals' diet is altered at a later age. (Pediatr Res 21: 347-351, 1987)
\end{abstract}

\section{Abbreviations}

HC, high cholesterol

TC, taurocholic acid

GC, glycocholic acid

rpm, revolutions per minute

C, Chow

Shortly after weaning, the uptake of fatty acids and cholesterol into the jejunum of rabbits is greater than in older animals (13 ). It is now recognized that the effect of diet is specific to different tissues, to different membranes and to varying ages or

Received February 24, 1986; accepted November 13, 1986.

Send requests for reprints to A. B. R. Thomson, Nutrition and Metabolism Research Group, Robert Newton Research Building, University of Alberta, Edmonton, Alberta, Canada T6G $2 \mathrm{C} 2$.

Supported by the Medical Research Council (Canada). stages in the animal's development (4). Complex changes occur in the form and function of the digestive tract as animals develop $(5,6)$ and it remains unclear by what mechanism(s) the intestine adapts (7). The reduced permeability of the intestine which occurs with aging is not due to major alterations in the mucosal surface area, although the perturbations in the lipid composition of the microvillus membrane may account for some of these changes in transport function $(8,9)$. Variations in dietary triacylglycerol saturation alter the lipid composition and fluidity of rat intestinal plasma membranes (10) and there is considerable evidence that other dietary factors may influence the intestinal absorption of nutrients (11). For example, in mature rabbits, feeding a diet low in cholesterol leads to an increase in the uptake of cholesterol and fatty acids $(12,13)$. In young and recently weaned rabbits, feeding a low-cholesterol diet alters the uptake of sugars and lipids (14), and some of these early changes in jejunal transport persist for at least 2 wk after the animals are returned to feeding with standard Purina Chow (St. Louis, MO). This study was undertaken in the growing rabbit to establish the effect of early feeding of an HC diet (2\%) on the jejunal, ileal, and colonic in vitro uptake of a homologous series of saturated fatty acids, dodecanol, cholesterol, and bile acids. We tested the hypothesis that early feeding with an $\mathrm{HC}$ diet alters the subsequent normal development of intestinal transport of lipids.

\section{METHODS}

Chemicals. Unlabeled dodecanol and fatty acids were obtained from Applied Science Laboratories Inc., State College, PA. Unlabeled octanoic acid, cholesterol, TC, and GC were all greater than $99 \%$ pure as supplied by Sigma. $\left[1-{ }^{14} \mathrm{C}\right]$-labeled octanoic acid (caprylic, 8:0) and stearic acid (18:0) were obtained from New England Nuclear, Boston, MA. $\left[1-{ }^{14} \mathrm{C}\right]$-labeled dodecanoic acid (lauric, 12:0) and myristic acid (14:0) were obtained from Applied Science Laboratories Inc. $\left[1-{ }^{14} \mathrm{C}\right]$-labeled GC and cholesterol were obtained from Amersham Canada Ltd., Oakville, Ontario, Canada. $\left[1-{ }^{14} \mathrm{C}\right]$-labeled TC, hexanoic acid (FA6:0), decanoic acid (capric, FA10:0), plamitc acid (FA16:0), dodecanol, and $\left[{ }^{3} \mathrm{H}\right]$-inulin were obtained from ICN Biomedical Inc., Montreal, Quebec, Canada. Inulin has an approximate molecular weight of 5000 and was used in all experiments as a nonpermeant marker of the adherent mucosal fluid volume.

Preparation of incubation solutions. The technique used for the preparation of the test solutions containing fatty acids, cholesterol, bile acids, and dodecanol has been published elsewhere $(15-17)$. For the preparation of the micellar solutions of cholesterol and fatty acids 14:0, 16:0, and 18:0, an appropriate amount of unlabeled probe molecule was dissolved in $150 \mathrm{ml}$ of Krebsbicarbonate buffer to yield final concentrations of $0.1 \mathrm{mM}$ cholesterol in $20 \mathrm{mM}$ taurodeoxycholic acid (18), and counts of $250,000 \mathrm{cpm}$ of ${ }^{3} \mathrm{H}$-inulin and $100,000 \mathrm{cpm}$ of ${ }^{14} \mathrm{C}$-labeled lipids. The concentration of taurocholic or glycocholic acids was $1 \mathrm{mM}$, 
the concentration of dodecanol was $0.2 \mathrm{mM}$, and the concentration of the fatty acids was FA 6:0 and 8:0, 1.0 mM; FA 10:0, 0.5 mM; FA 12:0,0.2 mM; FA 14:0, 0.5 mM; FA 16:0 0.5 mM; and FA 18:0, 0.1 mM.

Diet composition and feeding schedule. The standard pelleted Purina Rabbit Chow was modified to contain a $2 \%$ content of cholesterol (12). This was called HC and the standard Chow diet was considered to be the "normal" cholesterol diet. The HC and Chow diets were otherwise similar in their content of carbohydrate, protein, fat, vitamins, and minerals.

The female Dutch Belted rabbits were obtained at the age of 8 wk and were maintained on Purina Chow for 14 days. One group continued on Chow for a further 14 days, a second group was fed Chow for 14 days followed by $\mathrm{HC}$ for 10 days followed by the Chow diet for 4 days. A third group was fed Chow for 42 days, and a fourth group was fed Chow for 14 days, then $\mathrm{HC}$ for 10 days, followed by Chow for 18 days. The four groups were therefore fed as follows: 1) Chow, 28 days; 2) Chow, 14 days followed by $\mathrm{HC}$ for 10 days followed by Chow for 4 days $(\mathrm{HC})$; 3) Chow, 42 days, 4) Chow, 14 days, followed by HC for 10 days followed by Chow for 18 days (HC-C). The weight of the animals was determined twice weekly.

Tissue preparation. The rabbits were anesthetized with rhompin/ketamine and the intestinal uptake studies were performed. As outlined in detail elsewhere (16), short segments of proximal jejunum, distal ileum, and proximal colon were rapidly removed, rinsed with $150 \mathrm{ml}$ of cold saline, opened along the mesenteric border, and the mucosal surface was carefully washed with a stream of cold saline from a syringe to remove visible mucus and debris. Sheets of intestine were mounted in incubation chambers and clamped between two plastic plates so that the mucosal and serosal surfaces were exposed to separate incubation solutions with apertures in the plates exactly $1.0 \mathrm{~cm}$ in diameter. The chambers were transferred to beakers containing oxygenated Krebs-bicarbonate buffer at $37^{\circ} \mathrm{C}$ for a preincubation period of $10 \mathrm{~min}$. The chamber were then transferred to other beakers for specific experiments.

The preincubation and incubation solutions were mixed at identical stirring rates with circular magnetic bars, and the stirring rates were precisely adjusted by means of a strobe light. Stirring rates were reported as the rpm at which the stirring bar was driven. The bulk phase was stirred at $600 \mathrm{rpm}$ to reduce the effective thickness of the intestinal water layer (16). When the bulk phase is stirred, the unstirred layer resistance is low and intestinal uptake is greater than when the bulk phase is unstirred (16). The condition of stirring of the bulk phase was chosen to better demonstrate possible changes in transport function arising from dietary changes.

Determination of rates of uptake of fatty acids, bile acids, and cholesterol. After preincubation in Krebs-bicarbonate buffer for $10 \mathrm{~min}$, the chambers were transferred to other beakers containing $\left[{ }^{3} \mathrm{H}\right]$-inulin and various probes including $\left[{ }^{14} \mathrm{C}\right]$-labeled cho- lesterol, bile acids, and fatty acids in oxygenated Krebs-bicarbonate buffer at $37^{\circ} \mathrm{C}$. After incubation for $6 \mathrm{~min}$ in these test solutions, the experiment was terminated by removing the chamber and quickly rinsing the intestinal tissue in cold saline for approximately $5 \mathrm{~s}$. The exposed mucosal tissue was then cut out of the chamber with a circular steel punch, placed on glass slides, dried overnight in an oven at $55^{\circ} \mathrm{C}$, after which the dry weight was determined. The tissue was transferred to counting vials, saponified with $0.75 \mathrm{~N} \mathrm{NaOH}$, scintillation fluid was added, and radioactivity was determined by means of an external standardization technique to correct for variable quenching of the two isotopes.

Determination of rate of uptake of dodecanol. The rate of uptake of the diffusion-limited dodecanol was used to assess the effective resistance of the intestinal unstirred water layer $(16,23)$. The method used for the measurement of the rate of uptake of dodecanol was similar to that used for the lipids, except that the intestinal tissue was weighed wet and was placed in $0.7 \mathrm{ml}$ Protosol (New England Nuclear) at $55^{\circ} \mathrm{C}$ overnight (19). No loss of $\left[{ }^{14} \mathrm{C}\right]$-dodecanol was noted during incubation of the tissue at $55^{\circ} \mathrm{C}$ for periods of up to $36 \mathrm{~h}$. The samples were cooled to room temperature, scintillation fluid was added, and radioactivity was determined as described above. Separate pieces of jejunum were weighed wet and were reweighed after drying overnight at $55^{\circ} \mathrm{C}$. From the ratio of intestinal dry to wet weight, the rate of uptake of the dodecanol was changed from $\mathrm{nmol} / \mathrm{min} /$ $100 \mathrm{mg}$ wet weight to $\mathrm{nmol} / \mathrm{min} / 100 \mathrm{mg}$ dry weight. These latter units are similar to those used for the other solutes reported in this study.

Expression of results. The rate of uptake, $\mathrm{J}_{\mathrm{d}}$, was calculated after correcting the total tissue $\left[{ }^{14} \mathrm{C}\right]$-radioactivity for the mass of the probe molecule present in the adherent mucosal fluid. The uptake rates were expressed as the nmol of the probe molecule taken up into the mucosa per min per $100 \mathrm{mg}$ dry weight of tissue $(\mathrm{nmol} / \mathrm{min} / 100 \mathrm{mg})$. The values of the incremental change in free energy $\left(\int \Delta \mathrm{Fw} \rightarrow l\right)$ associated with the uptake of medium chain fatty acids (FA 6:0-12:0) were obtained after corrections made for unstirred layer resistance, as described previously $(2,3$, $16,23)$.

The values obtained for the different groups are reported as the mean \pm SEM of the results of six to nine animals. The statistical significance of the difference between any two means was determined by unpaired $t$ test: the values for $\mathrm{HC}$ were tested against Chow feeding for 28 days, and HC-C was compared with Chow feeding for 42 days.

Morphology. Morphometric measurements were done on 1$\mathrm{cm}$ sections of jejunum and ileum fixed in Bouin's solution, dehydrated, embedded in paraffin, sectioned for light microscopy, and stained with hematoxylin and eosin using standard techniques. The method of villus surface area calculation was done as previously reported (20). Villus height, villus width at $1 / 2$ height, villus bottom width, crypt depth, cell size, and the number

Table 1. Effect of $H C$ diet on characteristics of animals*

\begin{tabular}{|c|c|c|c|c|}
\hline & \multicolumn{2}{|c|}{ Chow } & \multirow[b]{2}{*}{$\mathrm{HC}$} & \multirow[b]{2}{*}{$\mathrm{HC}-\mathrm{C}$} \\
\hline & 28 Days & 42 Days & & \\
\hline Body wt gain (g/day) & $13.9 \pm 0.4$ & $15.5 \pm 0.7$ & $7.5 \pm 1 \dagger$ & $11.7 \pm 0.5 \dagger$ \\
\hline Wt at end of study (kg/animal) & $1.54 \pm 0.04$ & $1.74 \pm 0.02$ & $1.29 \pm 0.03 \dagger$ & $1.55 \pm 0.04 \uparrow$ \\
\hline \multicolumn{5}{|l|}{ Dry wt $(\mathrm{mg} / \mathrm{U})$} \\
\hline \multicolumn{5}{|l|}{ Serosal surface area } \\
\hline Jejunum & $11.2 \pm 0.6$ & $11.5 \pm 0.7$ & $10.5 \pm 0.7$ & $11.3 \pm 0.5$ \\
\hline Ileum & $10.6 \pm 0.5$ & $9.3 \pm 0.7$ & $9.9 \pm 0.5$ & $9.7 \pm 0.5$ \\
\hline Colon & $12.9 \pm 0.8$ & $9.3 \pm 0.6$ & $11.5 \pm 0.6$ & $10.8 \pm 0.6$ \\
\hline
\end{tabular}

${ }^{*} \mathrm{HC}$, Chow for 14 days followed by $\mathrm{HC}$ diet for 10 days followed by Chow for 4 days; HC-Chow, Chow for 14 days followed by HC diet for 10 days, followed by Chow for 18 days.

$\dagger p<0.05$, HC versus Chow feeding for 28 days, or HC-C versus Chow feeding for 42 days. 


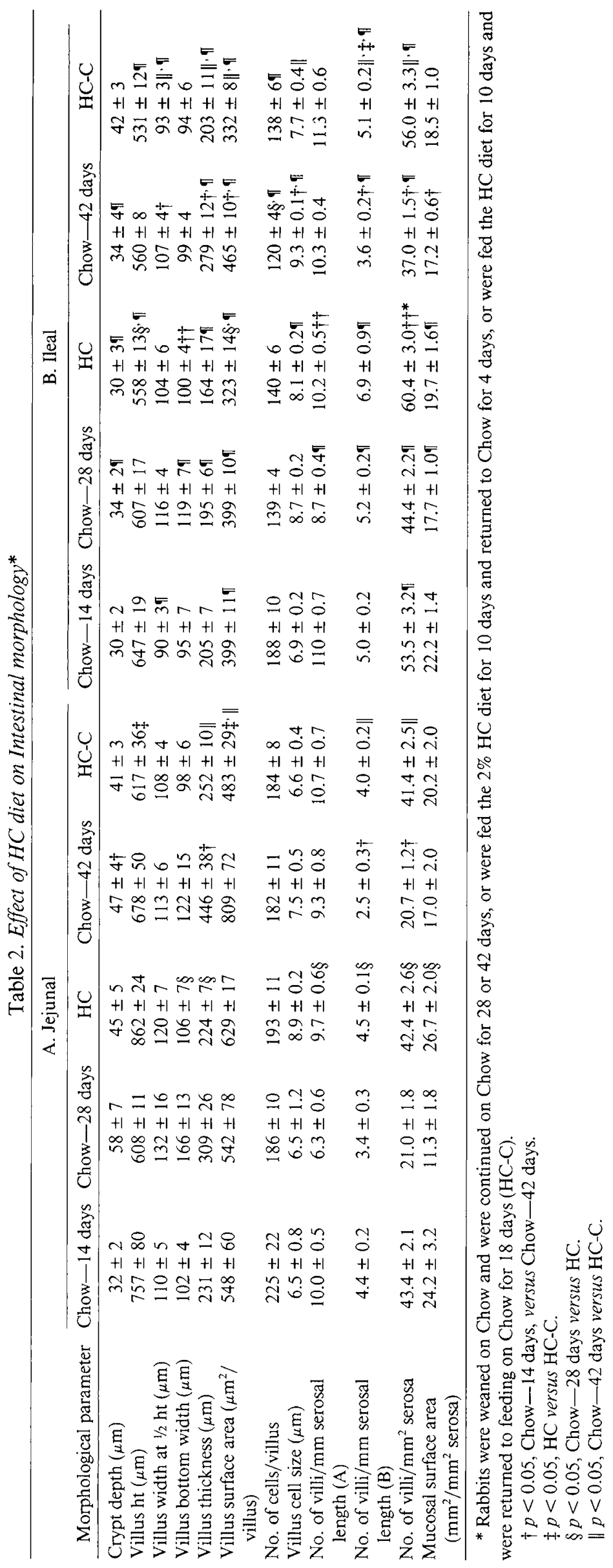

of cells per villus were determined from longitudinal sections. Horizontal cross-sections were prepared so that the second dimension of villus width, referred to here as villus thickness, could be measured at $1 / 2$ villus height. The density of the villi (number of villi/mm serosal length) was measured in longitudinal and horizontal cross-sections, then multiplied together to obtain the number of villi $/ \mathrm{mm}^{2}$ serosa. When this villus density was multiplied by the villus surface area, the result was the mucosal surface area, expressed as $\mathrm{mm}^{2} / \mathrm{mm}^{2}$ serosa. At least 10 villi were assessed per section. The following formulae were used:

Villus surface area $\left(\mu \mathrm{M}^{2} /\right.$ villus $)$

$$
\begin{aligned}
=(2 \times \mathrm{M} \times \mathrm{H})+(2 \times \mathrm{M}-\mathrm{A}) \times \mathrm{D}+ \\
(2 \times \mathrm{D}) \times\left[(\mathrm{A}-\mathrm{M})^{2}+(\mathrm{H})^{2}\right]^{0.5} \times 1000
\end{aligned}
$$

where $H=$ villus height, $M=$ villus width at $1 / 2$ height, $A=$ villus bottom width, $\mathrm{D}=$ villus thickness.

Mucosal surface area $\left(\mathrm{mm}^{2} / \mathrm{mm}^{2}\right.$ serosa)

$$
=\text { number of villi } / \mathrm{mm}^{2} \text { serosa }
$$

$$
\times \text { villus surface area }\left(\mathrm{mm}^{2} / \text { villus }\right)
$$

\section{RESULTS}

Characteristics of the animals. The animals were of similar weight and age at the outset. Body weight gain was less in HC and HC-C than when the animals were fed Chow for 28 or 42 days (Table 1). In HC-C, the animals were returned to Chow for 18 days following 10 days of the $\mathrm{HC}$ diet; they gained weight at the rate of $29.9 \pm 2.2 \mathrm{~g} /$ day, giving an average 42 day rate of weight gain of $11.7 \pm 0.5 \mathrm{~g} /$ day. These differences in body weight gain were due to the loss of $16 \pm 0.4 \mathrm{~g} /$ day while the animals were on HC. These differences in weight gain were associated with reduced food intake when the animals were fed the HC diet. Neither the dry weight nor the ratio of the dry/wet weight of the jejunum, ileum, or colon was affected by feeding $\mathrm{HC}$.

There were diet-related variations in some of the characteristics of the intestinal villi. Alterations in the cholesterol content of the

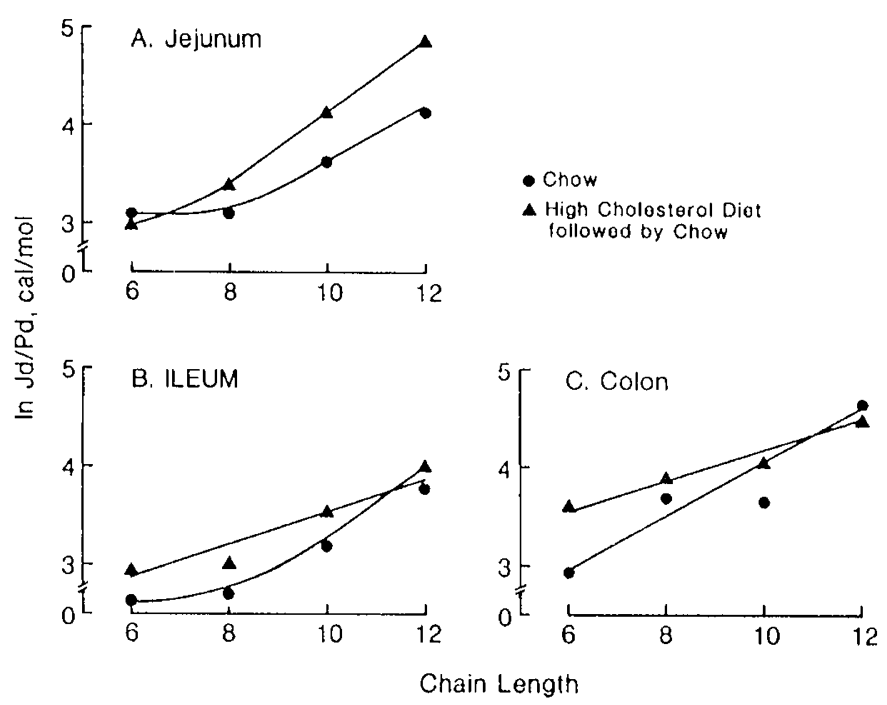

Fig. 1. Effect of early feeding of HC diet on the uptake of mediumchain fatty acids into jejunum, ileum, and colon. Rabbits were weaned on Chow and were continued on Chow or were fed $2 \% \mathrm{HC}$ diet for 10 days and returned to Chow for 4 days, or were fed $\mathrm{HC}$ for 10 days and were then returned to feeding on Chow for 18 days (HC-C). The values for $\mathrm{HC}$ were similar to Chow and are not shown. The chain length of the fatty acids is plotted versus the natural logarithm of the rate of uptake for $\mathrm{mM}$ divided by the appropriate aqueous diffusion coefficient. The slope was greater in HC-C than in Chow-fed animals in the jejunum but was lower in the ileum and colon. 
diet had no effect on the depth of the crypts in the jejunum or ileum (Table $2 a$ ). Feeding HC was associated with an increase in the height of the jejunal villi and the jejunal mucosal surface area as compared to animals fed Chow for 28 days. In the ileum (Table $2 B$ ), feeding $\mathrm{HC}$ was associated with a fall in villus height and villus surface area but an unchanged mucosal surface area (due to an increase in villus density, not shown). Switching from $\mathrm{HC}$ to Chow for 18 days (HC-C) was associated with a decline in jejunal but not ileal villus height, and villus and mucosal surface area $(p<0.05)$ when compared to animals fed $\mathrm{HC}$.

Fatty acids and cholesterol. Feeding HC-C was associated with increased $(p<0.05)$ jejunal uptake of FA 8:0, 10;0, and 12:0 (Fig. 1), leading to a greater value for the slope of the relationship between the number of $\mathrm{CH}_{2}$ units in the fatty acid chain, versus the natural logarithm of uptake divided by the appropriate aqueous diffusion coefficient (In Jd/Pd). In contrast, HC-C was

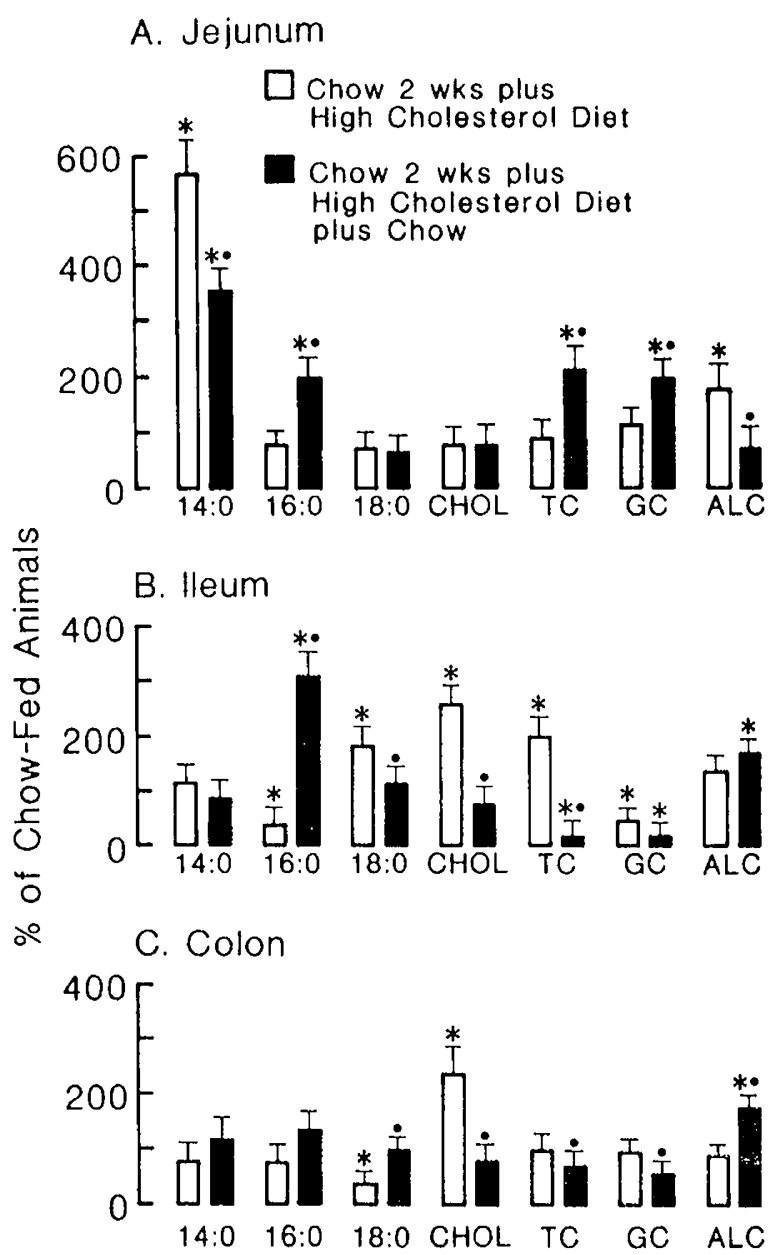

Fig. 2. Effect of early feeding of $\mathrm{HC}$ diet on the jejunal uptake of fatty acids, cholesterol, bile acids, and dodecanol into the jejunum, ileum, and colon. Rabbits were weaned on Chow and were continued on Chow or were fed the $2 \% \mathrm{HC}$ diet for 10 days and returned to Chow for 4 days, or were fed $\mathrm{HC}$ for 10 days and were then returned to feeding on Chow for 18 days (HC-C). The height of the bars represents the mean \pm SEM of the results of six to nine animals. An asterisk indicates that the difference between the mean value of the group fed $\mathrm{HC}$ was statistically significant from the group fed Chow for 28 days, or that the difference between the mean value of the group fed $\mathrm{HC}-\mathrm{C}$ was statistically different from the group fed Chow for 42 days $(p<0.05)$. A dot indicates that the difference between the mean values of $\mathrm{HC}-\mathrm{C}$ versus $\mathrm{HC}$ was statistically significant $(p<0.05)$. The number abbreviations represent the chain length of the saturated fatty acids (e.g. 14:0 is myristic acid, 16:0 is palmitic acid, and 18:0 is stearic acid). CHOL, cholesterol; $A L C$, dodecanol. not associated with any change in the value of $\int^{\Delta} \mathrm{Fw} \rightarrow l$ for the uptake of medium-chain fatty acids into the ileum or colon. There were no consistent effects of $\mathrm{HC}$ on the value of $\int^{\Delta} \mathrm{Fw} \rightarrow 1$ (not shown).

In the jejunum, the uptake of myristic acid and dodecanol was greater in HC compared with rabbits fed Chow (Fig. 2), whereas in the ileum, feeding $\mathrm{HC}$ was associated with increased uptake of stearic acid, cholesterol, and taurocholic acid but reduced uptake of glycocholic acid. In the colon, the uptake of stearic acid was lower but the uptake of cholesterol was higher in $\mathrm{HC}$ than in Chow-fed animals.

In the jejunum, the uptake of myristic acid and dodecanol was lower in $\mathrm{HC}-\mathrm{C}$ as compared with $\mathrm{HC}$, whereas there was increased uptake of palmitic acid, TC, and GC. In the ileum, the uptake of palmitic acid was higher in HC-C than in HC, but the uptake of stearic acid, cholesterol, TC, and GC was reduced. In the colon, the uptake of stearic acid and dodecanol was higher in $\mathrm{HC}-\mathrm{C}$ than in $\mathrm{HC}$, but in contrast, the uptake of cholesterol, $\mathrm{TC}$, and GC was lower.

\section{DISCUSSION}

The changes in lipid uptake were not simply explained by alterations in the morphology of the intestine: the mucosal surface area of the ileum was unaltered by diet (Table 2) even though there were diet-related changes in the uptake of lipids (Figs. 1 and 2). Furthermore, in the jejunum the mucosal surface area was higher in $\mathrm{HC}$ then in Chow-fed animals, similar in $\mathrm{HC}$ $\mathrm{C}$ and Chow-fed animals, yet lower in HC-C than in HC. In addition, the jejunal uptake of several lipids was similar in $\mathrm{HC}$ and in Chowd animals and greater in HC-C than in Chow-fed animals. Thus, mucosal surface area did vary between 14-42 days of feeding Chow, was increased by feeding HC, but was not the simple explanation for alterations in intestinal uptake.

The second barrier to intestinal permeation is the effective resistance of the intestinal unstirred water layer $(23,24)$. This resistance may be assessed from the rate of uptake of dodecanol (16). The feeding of HC was associated with an increased jejunal uptake of dodecanol and therefore a decreased effective resistance of the unstirred water layer. In contrast, unstirred layer resistance declined 2 wk after switching from the HC diet back HC-C. Thus, although the effective resistance of the unstirred layer varied in response to dietary manipulations, this was not invariably associated with appropriate changes in the uptake of the lipids (Figs. 1 and 2) and there must be some other explanation for the alterations in lipid uptake.

Dietary changes may alter the microviscosity and lipid composition of the intestinal brush border membrane $(10,21)$. However, diet-related changes in intestinal transport function are not necessarily associated with changes in the membrane content of either cholesterol or phospholipids (21). Furthermore, changes in intestinal lipid composition are not necessarily associated with changes in membrane fluidity (22). Aging itself alters the lipid composition of the brush border membrane (9). The effect of short-term feeding of a $\mathrm{HC} \operatorname{diet}(2 \%)$ on brush border membrane composition has not yet been reported. In view of the increased jejunal permeability to lipids in $\mathrm{HC}-\mathrm{C}\left(\int^{\Delta} \mathrm{Fw} \rightarrow \mathrm{l}\right)$, it is speculated that this diet might alter the lipid composition of the brush border membrane, thereby influencing the in vitro uptake of medium-chain fatty acids observed in animals fed this diet (Fig. 2).

In the jejunum, HC-C was associated with increased uptake of FA 8:0-18:0 but not cholesterol or bile acids, whereas in the ileum and colon HC-C was associated with decreased $\int{ }^{\Delta} \mathrm{Fw} \rightarrow 1$ and increased uptake of only FA 18:0. This different pattern noted between the proximal and distal intestine may simply reflect the length along the intestine where the influence of the dietary signal is expressed or a difference in the manner of response of the jejunum versus the ileum. The enhancement of bile acid uptake into the ileum but not into the jejunum or colon of $\mathrm{HC}-\mathrm{C}$ may reflect the differences in the mechanisms of trans- 
port, active in the ileum and passive in the jejunum and colon. Thus, dietary manipulation has a differential effect on the intestinal transport of lipids and this effect may become more marked after the dietary challenge has been withdrawn.

Much has been written on the effect of cholesterol in human milk (29). This study did not address the question of the relative merits of this practise. However, the effects of feeding the $\mathrm{HC}$ diet became most pronounced (Figs. 1 and 2) when the animals were switched back to Chow (HC-C). Thus, the effects of early feeding experiences may become manifest only at a later age or when the initial dietary challenge has been removed. The nature of the diet fed to young and developing animals may have a pronounced effect on the membrane composition and function of organs including brain, heart, and liver $(27,28)$. For these reasons, and since early feeding experiences may alter the subsequent normal development of the permeability properties of the intestine, the late effects of early dietary changes must be carefully examined before alterations in early feeding experiences are widely recommended.

Acknowledgments. The authors thank Susan Evans-Davies for her secretarial assistance, and Laura Poland, Monica Mutual, Anne Williams, Doris Luethe, Kathy Kirdeikis for their expert technical assistance, and the Alberta Heritage Foundation for Medical Research for assistance in the purchase of major equipment in the conduct of this study.

\section{REFERENCES}

1. Thomson ABR 1979 Unstirred water layer and age-dependent changes in rabbit jejunal D-glucose transport. Am J Physiol 5:E685-E691

2. Thomson ABR 1980 Effect of age on uptake of homologous series of saturated fatty acids into rabbit jejunum. Am J Physiol 239:G363-G371

3. Thomson ABR 1981 Ageing and cholesterol uptake in the rabbit jejunum. Dig Dis Sci 26:890-896

4. Clandinin MT, Field CJ, Hargreaves K, Morson LA, Zsigmond E 1985 Role of diet in subcellular structure and function. CFBS Symposium Lecture. Can J Physiol Pharmacol 63:546-556

5. Thomson ABR, Keelan M 1986a The development of the small intestine. Can J Physiol Pharmacol 64:13-29

6. Thomson ABR, Keelan M 1986b The aging intestine. Can J Physiol Pharmacol 64:30-38

7. Karasov WH, Diamond JH 1983 Adaptive regulation of sugar and amino acid transport by vertebrate intestine. Am J Physiol 8:G443-G462

8. Brasitus TA, Yeh K-Y, Holt PR, Schachter D 1984 Lipid fluidity and composition of intestinal microvillus membranes isolated from rats of different ages. Biochim Biophysica Acta 778:341-348

9. Keelan M, Walker K, Thomson ABR 1985 Intestinal morphology, marker enzymes and lipid content of brush border membranes from rabbit jejunum and ileum: effect of aging. Mech Aging Dev 31:49-68

10. Brasitus TA, Davidson NO, Schachter D 1985 Variations in dietary triacylglycerol saturation alter the lipid composition and fluidity of rat intestinal plasma membranes. Biochim Biophysica Acta 812:460-472

11. Thomson $A B R$, Keelan M 1985c Effect of oral nutrition on the form and function of the intestinal tract. Surg Dig Dis 3:75-94

12. Thomson ABR 1982 Influence of dietary modifications on uptake of cholesterol, glucose, fatty acids and alcohols into rabbit intestine. Am $\mathrm{J}$ Clin Nutr 35:556-565

13. Thomson ABR, Rajotte R 1983 Effect of dietary modification on the enhanced uptake of cholesterol in diabetic rats. Am J Clin Nutr 37:394-403

14. Thomson ABR 1986 Early nutrition and intestinal transport function: effect of a low-cholesterol diet. J Lab Clin Med 107:365-377

15. Lukie BE, Westergaard H, Dietschy JM 1974 Validation of a chamber that allows measurement of both tissue uptake rates and unstirred layer thickness in the intestine under conditions of controlled stirring. Gastroenterology 67:652-661

16. Westergaard H, Dietschy JM 1974 Delineation of the dimensions and permeability characteristics of the two major diffusion barriers to passive mucosal uptake in the rabbit intestine. $J$ Clin Invest $54: 718-732$

17. Thomson ABR, Dietschy JM 1980 Experimental demonstration of the effect of the unstirred water layer on the kinetic constants of the membrane transport process for D-glucose in rabbit jejunum. J Memb Biol 54:221-229

18. Westergaard H, Dietschy JM 1976 The mechanisms whereby bile acid micelles increase the rate of fatty acid and cholesterol uptake into the intestinal mucosal cell. J Clin Invest 58:97-108

19. Thomson ABR $1980 \mathrm{~b}$ Unidirectional flux rte of cholesterol and fatty acids into the intestine of rats with drug-induced diabetes mellitus: effect of variations in the effective resistance of the unstirred water layer and the bile acid micelle. J Lipid Res 21:687-698

20. Ecknauer R, Vadakel T, Wepler R 1982 Intestinal morphology and cell production rate in aging rats. $\mathrm{J}$ Gerontol 37:151-155

21. Thomson ABR, Keelan M, Clandinin MT, Walker K 1986 Dietary fat selectively alters transport properties of rat jejunum. J Clin Invest 77:279-288

22. Di Costanza G, Duportail G, Florentz A, Leroy C 1983 The brush border membrane of trout intestine: influence of its lipid composition on ion permeability, enzyme activity and membrane fluidity. Mol Physiol 4:279_ 290

23. Thomson ABR, Dietschy JM 1984 The role of the unstirred water layer in intestinal permeation. In: Csakyi TZ (ed) Pharmacology of Intestinal Permeation. Springer-Verlag, Berlin, pp 165-269

24. Barry PH, Diamond JM 1984 Effects of unstirred layers on membrane phenomena. Physiol Rev 64:763-872

25. Winne D 1978 The permeability coefficient of the wall of a villus mebrane. $J$ Math Biol 6:9-108

26. Morin CL, Grey VL, Garofalo C 1982 In: Robinson JWL, Dowling RH, Riecken EO (eds) Mechanisms of Intestinal Adaptation. MTP Press Ltd, Boston, pp 175-184

27. Innis SM, Clandinin MT 1981 Dynamic modulation of mitochondrial innermembrane lipids in rat heart by dietary fat. Biochim J 193:155-167

28. Innis SM, Clandinin MT 1982 Mitochondrial membrane polar-head-group composition is influenced by diet fat. Biochim J 198:231-234

29. Clandinin MT, Chappell JE, Hargreaves K, Thomson ABR 1987 The effect of diet fat in milk and tissue lipids. AOCS Monogr (in press) 